\title{
QUEEN'S
UNIVERSITY
BELFAST
}

\section{A novel experimental approach to investigate radiolysis processes in liquid samples using collimated radiation sources}

Polin, C., Wardlow, N., McQuaid, H., Orr, P., Villagomez-Bernabe, B., Figueira, C., Alexander, G., Srigengan, S., Brun, E., Gilles, M., Sicard-Roselli, C., \& Currell, F. J. (2015). A novel experimental approach to investigate radiolysis processes in liquid samples using collimated radiation sources. Review of Scientific Instruments, 86(3), [035106]. https://doi.org/10.1063/1.4914054

Published in:

Review of Scientific Instruments

Document Version:

Peer reviewed version

Queen's University Belfast - Research Portal:

Link to publication record in Queen's University Belfast Research Portal

Publisher rights

(C) 2015 AIP Publishing LLC.

Reprinted with permission from Review of Scientific Instruments, American Institute of Physics.

\section{General rights}

Copyright for the publications made accessible via the Queen's University Belfast Research Portal is retained by the author(s) and / or other copyright owners and it is a condition of accessing these publications that users recognise and abide by the legal requirements associated with these rights.

Take down policy

The Research Portal is Queen's institutional repository that provides access to Queen's research output. Every effort has been made to ensure that content in the Research Portal does not infringe any person's rights, or applicable UK laws. If you discover content in the Research Portal that you believe breaches copyright or violates any law, please contact openaccess@qub.ac.uk. 


\title{
A NOVEL EXPERIMENTAL APPROACH TO INVESTIGATE RADIOLYSIS PROCESSES IN LIQUID SAMPLES USING COLLIMATED RADIATION SOURCES.
}

\author{
Chris Polin, ${ }^{1, a)}$ Nathan Wardlow, ${ }^{1}$ Harold McQuaid, ${ }^{1}$ Philip Orr, ${ }^{1}$ Balder Villagomez-Bernabe, ${ }^{1}$ Catarina \\ Figueira, ${ }^{1}$ Grace Alexander, ${ }^{1}$ Shajeth Srigengan, ${ }^{2}$ Emilie Brun, ${ }^{3}$ Manon Gilles, ${ }^{3}$ Cécile Sicard-Roselli, ${ }^{3}$ and Fred J. \\ Currell ${ }^{1}$ \\ 1) Centre for Plasma Physics, Department of Mathematics and Physics, Queen's University Belfast \\ 2) School of Physics and Astronomy, University of Manchester \\ ${ }^{3)}$ Laboratoire de Chimie Physique, Université Paris-Sud
}

(Dated: 17 February 2015)

Here is detailed a novel and low-cost experimental method for high-throughput automated fluid sample irradiation. The sample is delivered via syringe pump to a nozzle, where it is expressed in the form of a hanging droplet into the path of a beam of ionising radiation. The dose delivery is controlled by an upstream lead shutter, which allows the beam to reach the droplet for a user defined period of time. The droplet is then further expressed after irradiation until it falls into one well of a standard microplate. The entire system is automated and can be operated remotely using software designed in-house, allowing for use in environments deemed unsafe for the user (synchrotron beamlines, for example). Depending on the number of wells in the microplate, several droplets can be irradiated before any human interaction is necessary, and the user may choose up to 10 samples per microplate using an array of identical syringe pumps, the design of which is described here. The nozzles consistently produce droplets of $25.1 \pm 0.5 \mu l$.

\section{INTRODUCTION AND BACKGROUND}

Radiolysis is a widely used experimental technique, providing the chemical basis for the description of a num- ber of applications including cancer radiotherapy ${ }^{1}$, civil nuclear power ${ }^{2}$, and the semiconductor industry ${ }^{3}$.

The initial impetus behind the development

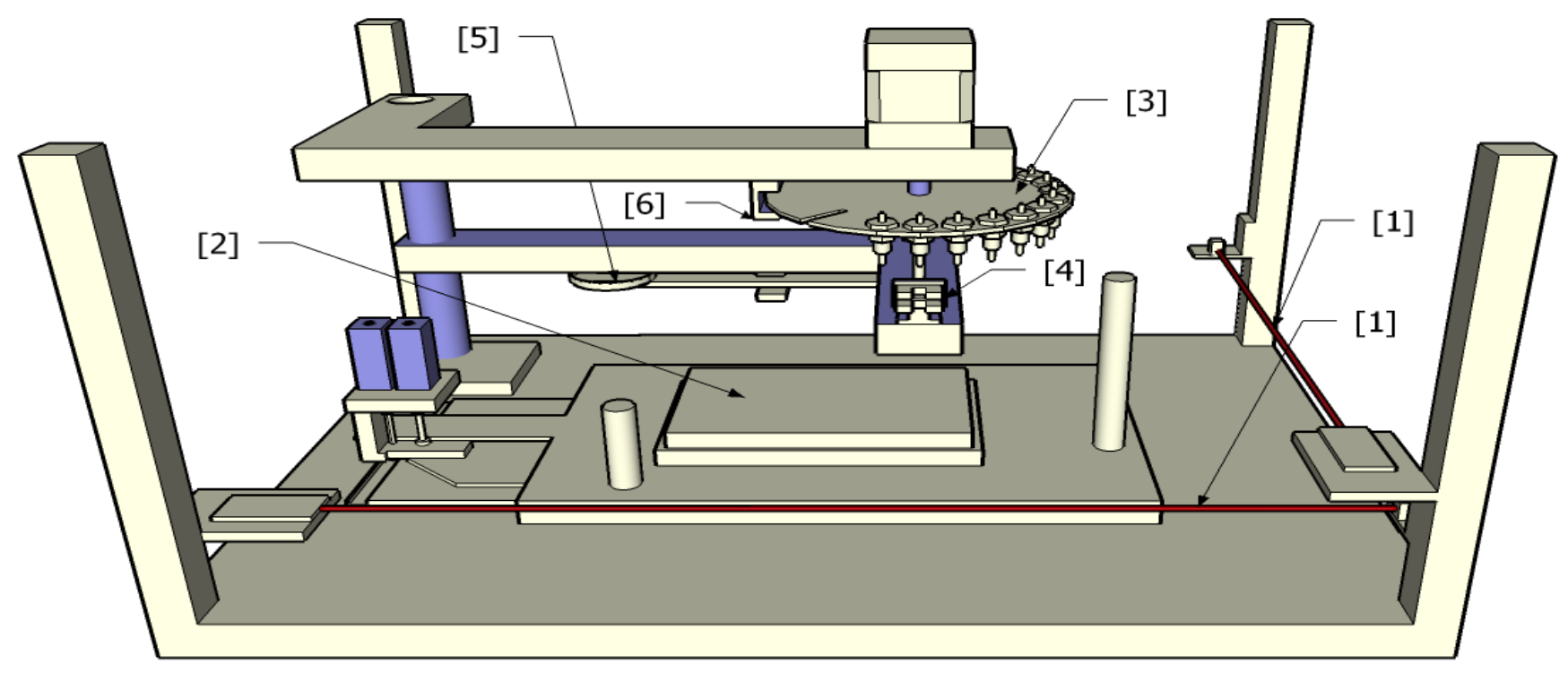

FIG. 1. Schematic diagram of the apparatus with the main components detailed: [1] Stage Datum Laser Gate, [2] Multiwell Microplate, [3] Nozzle Carousel, [4] Droplet Detector, [5] Waste Arm, [6] Carousel Datum.

\footnotetext{
a) Electronic mail: cpolin01@qub.ac.uk
}

of the apparatus described here was to create an automated, high throughput system which could directly subject fluid samples to a collimated beam of ionizing radiation using a highly automated 'hanging droplet' arrangement (removing the necessity of a container).

It was designed specifically as part of an end-station for a synchrotron beam, although it can be adapted to any beam setup capable of supporting it. 


\section{DESCRIPTION OF APPARATUS}

A schematic overview of the apparatus is shown in Figure 1. A droplet is expressed from a nozzle through the use of a syringe pump to the point where it is just about to fall. The droplet is then irradiated for a set period of time through the use of an upstream computer controlled radiation shutter. Once this operation is complete, the droplet is further expressed until it falls into a multiwell plate situated directly below the nozzle. The multiwell plate is mounted on an automated $\mathrm{x}-\mathrm{y}$ translator, so each well in turn can be filled with an irradiated sample through repeated application of the procedure described above. Under the standard configuration, the system accepts 96-well microplates and as it is entirely automated and controlled remotely using software designed and developed in house, it is capable of exposing up to 96 samples to a beam before any human interaction is required. It is capable of accepting other sized microplates and can be programmed to accommodate them.

In practice, repeated irradiation of a single sample type is somewhat restrictive, so an array of ten nozzles is mounted on a carousel, with each nozzle being controlled by a separate syringe pump. The carousel can be rotated under computer control so that any of the ten nozzles is positioned directly above the path of the radiation beam. In this way, any one of ten different samples can be irradiated as required.

This system negates the issue of potential container effects as the samples are suspended as droplets into the path of a horizontally oriented beam. This is a very beneficial feature in many applications; for example when using low-penetration beams, there is no concern of attenuation before the sample (save for the air kerma between the source and the droplet). In addition, this way a precisely replicable volume of the sample is irradiated.

Due to the automated nature of the apparatus, it is ideal for use in environments where human interaction would be hazardous, such as synchrotron beamlines, ion accelerators or LINACs. It is relatively compact (540 $\mathrm{mm} \times 330 \mathrm{~mm}$ x $410 \mathrm{~mm}$ ) and can be integrated easily with other equipment as the experiment requires.

The apparatus consists of three main components: The sample delivery, lead shutter and sample collection systems. Each has been incorporated into an automated protocol and work in sequence to produce a consistently sized droplet from a nozzle, irradiate it with a reproducible dose, and deposit it in the plate below for easy analysis.

\section{A. Operational Protocol}

The system has been designed to maximise versitility, and as such is entirely programmable to the user's requirements. The hardware itself is capable of translating directly and rapidly to any well in the microplate and placing one or several droplets into it, with or without

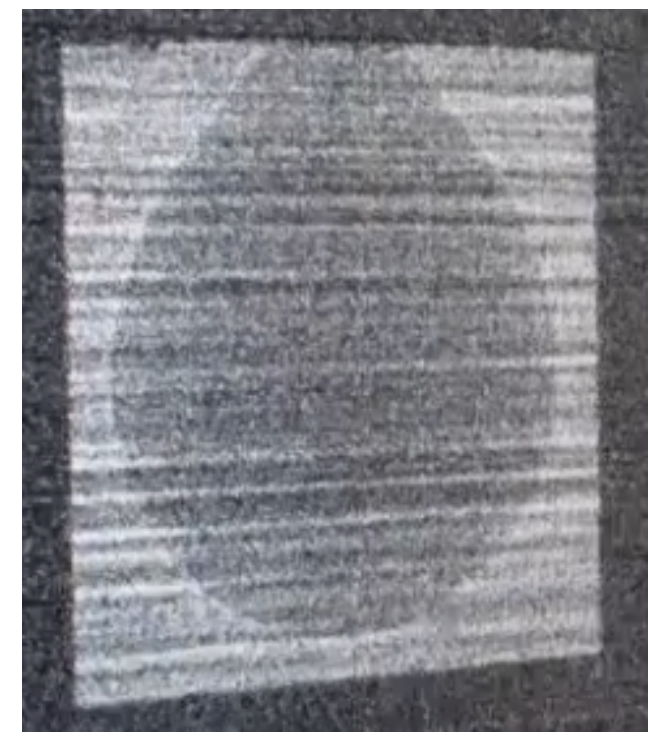

FIG. 2. This image was taken from an x-ray camera situated in the beam path downstream from the apparatus. A droplet can clearly be seen attenuating the beam. It should be noted that the ostensible skew in the beam profile is as a result of the camera's angle with respect to the beam axis.

exposing them to a beam. It can be configured to act on a timer or any external trigger capable of sending digital logic signals.

While the device can be adapted to any experiment, it has been designed and built with a specific operational protocol in mind, which is outlined in Figure 3.

In the initialisation phase of the protocol, the user begins by entering a plate plan into the control program, assigning an irradiation dose and sample to each well of the microplate. The $\mathrm{x}-\mathrm{y}$ stage is positioned by the software so that the first well in the microplate is directly beneath the nozzle. The syringes and lines are loaded with the desired samples.

From this point, the process is automated and requires no further user input. The software reads the syringe number assigned to the current well and rotates the carousel until the desired nozzle is in position above the beam path.

The syringe is then depressed with a predetermined amount of motor steps (this value is produced in a separate calibration program and saved to file) so that a droplet is expressed and suspended from the nozzle tip into the beam path.

The shutter opens for a predetermined period of time depending on the value entered into the plate plan, allowing the beam to irradiate the sample with a specific dose. Once this period of time elapses, the shutter closes.

After irradiation, the syringe is depressed further to expel the droplet from the nozzle into the well. The droplet falls through an opto-interrupter before it reaches the multiwell plate, alerting the software to deactivate the syringe motor. 


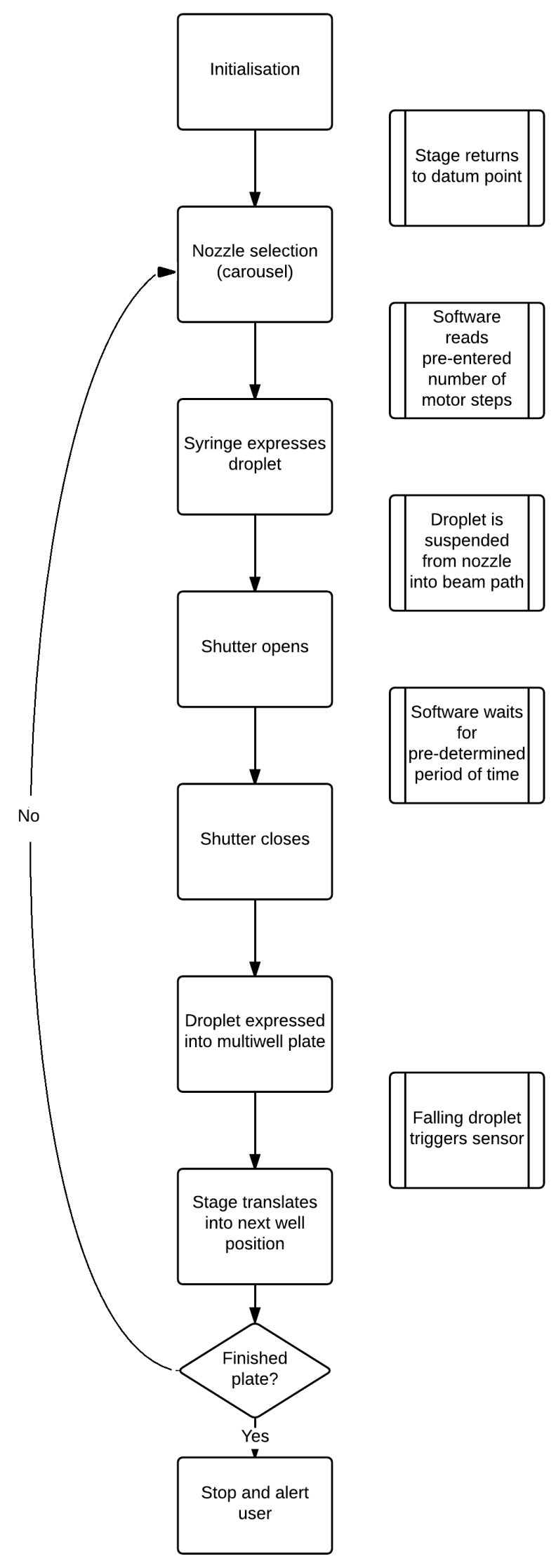

FIG. 3. The operational protocol of the system software.

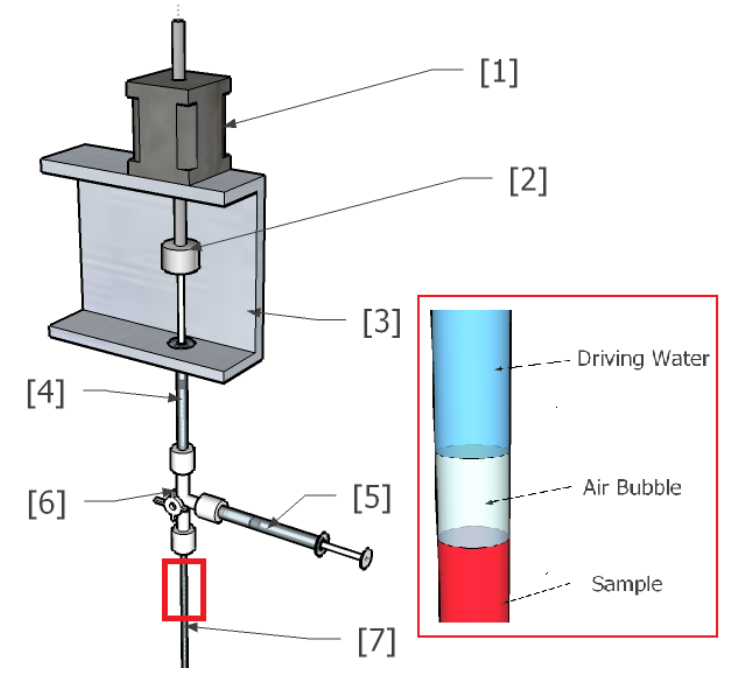

FIG. 4. A rendering of the syringe pump design. The linear actuator [1] is connected to the syringe plunger with an adaptor [2], allowing it to both push and pull. A 'C'-section support beam [3] holds the actuator above the driver syringe [4]. The secondary 'reservoir' syringe [5] is also shown, attached to a 3-way stopcock [6]. The sample is then delivered along a PTFE tube [7]. Note the air bubble between the driving water and sample interface, highlighted. The diagram shows one full syringe pump, but the design can be replicated up to 15 times on a 16-channel multiplexor IC.

Once the droplet has been detected as having fallen, the stage then translates so the next well is positioned beneath the nozzle, and the process repeats for the next sample. The standard programming dictates that the wells are filled one row at a time, although it is possible to skip wells and rows if desired.

\section{B. Sample Delivery System}

We have developed a low-cost, automated microlitreprecision syringe pump which can be replicated into an array if required.

Samples are expressed as droplets from an inert polyetheretherketone (PEEK) nozzle (coleparmer.com, 0.010in bore Polyetheretherketone chromatography tubing, product code: EW-02006-00). The nozzles are attached to narrow-bore (altecweb.com, 1/32 in bore PTFE tubing, product code: 01-96-1701) PTFE chromatography tubing, which terminates at a syringe pump. The syringe pump design consists of a linear actuator stepper motor (Zapp Automation SY17STH48A6.35-100CA, driven by a UIM24002 driver chip), a standard $1 \mathrm{ml}$ luerlock syringe with an attachment to connect the plunger to the shaft of the stepper motor, and an aluminium ' $\mathrm{C}$ 'section beam to hold the components. The system can also easily accommodate larger (up to $5 \mathrm{ml}$ ) syringes but in this case the precision of delivery is reduced since each motor step corresponds to a bigger volume displacement. 
The driver syringe is attached to a 3-way stopcock, which in turn leads to the PTFE tubing to the nozzle. The third port on the stopcock holds a secondary reservoir syringe, which is used for recharging the line. The driving syringe is filled with water, and the sample is then placed into a shallow receptacle and manually drawn up from the nozzle end of the PTFE tubing using the reservoir syringe. By filling the line with the sample from the nozzle upwards, it is possible to introduce a small bubble of air between the sample and driving water, thus ensuring that they do not mix and dilute the sample (see Figure 4). The bubble procedure is not necessary but is useful when the sample to be irradiated is valuable or in limited supply as it allows only the required volume of sample to be used and results in the minimum possible dead space and sample wastage. The driving water acts to be virtually incompressible, allowing for reliable droplet volume consistency.

In order to increase throughput and allow for ease of switching between samples, the design described above is replicated several times to form an array of syringe pumps. The entire array is then mounted above the rest of the apparatus. Individual syringes are selected via a multiplexor IC (Texas Instruments CD74HCT154E).

Extensive characterisation of the nozzles has been carried out in order to ensure droplet reproducibility. Tests were conducted measuring droplet volume (by mass of water) from nozzles tapered at $30^{\circ}, 45^{\circ}, 60^{\circ}$ and $90^{\circ}$ (untapered). As expected, the volume of the droplets upon falling from the nozzle decreased as the angle of the taper became sharper, due to reduced surface contact area. For all taper angles, the deviation of droplet size over a series of 30 consecutive droplets was under $2 \%$.

However, at sharper angles the process of droplet detachment became unpredictable, occasionally imparting a transverse component of motion and swinging the droplet outwards from the tip of the nozzle. This resulted in the droplets missing the wells of the microplate, and so a $45^{\circ}$ nozzle was decided upon. These produce a droplet volume of $25.1 \pm 0.5 \mu l$.

It should be noted that it is possible to further reduce the size of the droplets by decreasing the diameter of the nozzle. The syringe pumps can be used to both express and draw fluid. The system has been characterised solely in the 'push' mode and no attempt has been made to minimise hysteresis as it does not matter in this application.

\section{Lead Shutter System}

A lead shutter has been developed, to mount upstream from the apparatus. The shutter consists of a $1.8 \mathrm{~mm}$ thick lead plate with a window to permit the beam to pass when it is in the open position. A pair of opposing solenoids provide the force required to open and close the shutter, sliding the window in and out of the beam path as and when required. The shutter is mounted separately from the apparatus due to the possibility of vibrations travelling through to the droplets and dislodging them prematurely.

The $1.8 \mathrm{~mm}$ lead plate is capable of attenuating X-rays of up to $80 \mathrm{keV}$ down to $1 \%$ (according to information from the NIST database ${ }^{4}$ ).

The irradiation dose to each sample can be controlled and varied per droplet, allowing for entire dose ranges to be studied together in a single microplate. A 'plate plan' is entered into the user interface before a plate is run in the apparatus. The plan assigns a syringe number and a dose (in Gy) to each of the individual wells in the microplate. Specific dose is achieved by determining the dose rate at the droplet (this can be achieved by using chemical dosimetry, calibrated diode calculations, or other means) which is then converted to irradiation period. The upstream shutter then opens for the specified period, and the droplet is irradiated with the chosen dose.

\section{Sample Collection System}

Once the droplets have been irradiated, they fall into a multiwell microplate below. The plate is secured in a perspex holder attached to an x-y translator. At the beginning of an irradiation cycle, the first well is positioned directly below the active nozzle. After each droplet falls into a well in the plate, the translator moves so that the next well is in position, and the entire protocol repeats.

For the software to accurately align the microplate under the droplet nozzle, it is imperative that it has a fiducial point from which it can operate. This point is defined by two optical sensors running perpendicular to one another, and parallel to the axes of the stage's movement. Each of these sensor systems comprises a laser beam directed into a phototransistor at the opposite end of the apparatus and a vertical post (see Figure 5). When the stage reaches a certain position, the vertical post breaks the beam and prevents it from reaching the phototransistor. When this happens, the transistor raises the voltage on its output pin from $\sim 0$ to the input voltage $(5 \mathrm{~V})$. This is interpreted by the software as a logic 'true', and the motor corresponding to that axis halts. When both beams have been cut, the stage is in position.

\section{E. Peripheral Hardware}

Aside from the main parts of the apparatus, there are a number of components required for the apparatus to interface with the corresponding software.

\section{Droplet Detector}

The system must have a means of determining whether a droplet has fallen from the nozzle. To this end, a de- 


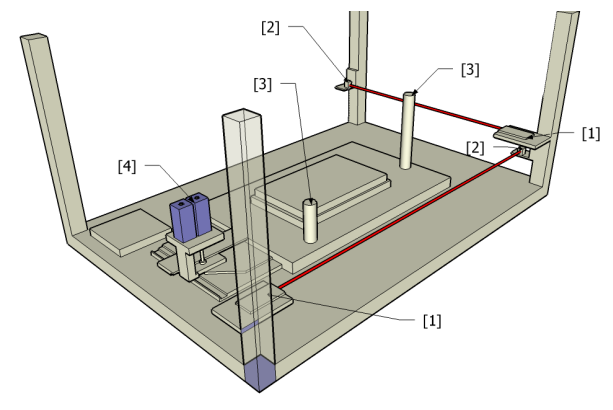

FIG. 5. Here a model of the translation datum mechanism can be seen. The two lasers [1] are directed into phototransistors [2], and they are interrupted by the pillars [3] on the translating stage. The stage itself is driven automatically using stepper motors [4].

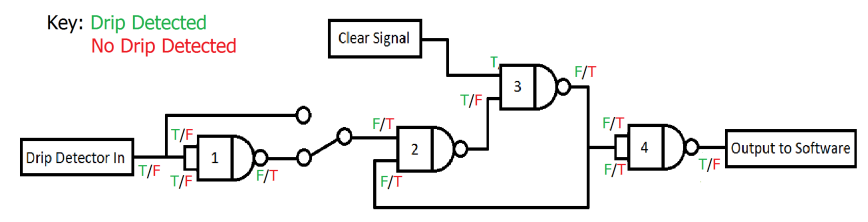

FIG. 6. The logic circuit accompanying the droplet detector.

tector was installed between the nozzle and the plate. The detector consists of a photo-coupler (Omron EESX4070) in line with the droplet's path, and a pull-up resistor. The photo-couplers are ' $\mathrm{C}$ '-shaped, with an infrared LED and a photo transistor facing one another. As the droplet falls between the emitter and detector, the light is cut and the signal pulses from $\sim 0$ to the input voltage. The NAND logic in the circuit (gates 2 and 3 of Figure 6 ) retains the true state until the computer polls the droplet circuit, so as to avoid missing droplets, thereby freeing the processor up for other tasks rather than continually polling the droplet detector line. The first NAND gate provides optional inversion of the signal in case EE-SX3070s are being used instead of EE-SX4070s. In practice, two photo-couplers and corresponding logic are employed here to increase the error tolerance, reducing the chance of an undetected droplet.

\section{Waste Arm}

A retractable arm with a receptacle to catch waste droplets is positioned between the droplet nozzle and the multiwell microplate. This is useful for when a line is being purged to change sample, or during the initial syringe calibration program where three droplets are expelled from the nozzle. The arm is attached to a stepper motor, and can be moved into position or retracted automatically as and when required. Another EE-SX4070 optical light gate is used to signal to the software when the arm is fully retracted.

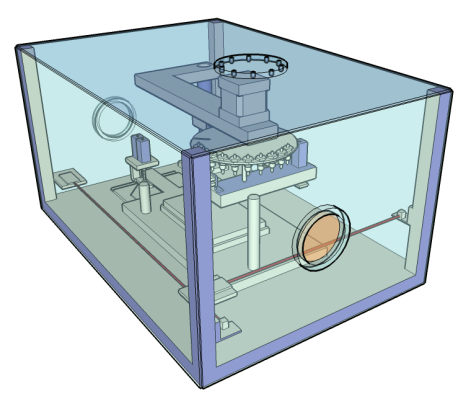

FIG. 7. The device is contained entirely within a perspex box, to allow the atmosphere where the droplets are irradiated to be controlled. Note the Kapton windows where the beam enters and exits the box, and the inlets on the top for the PTFE tubing which carries the samples.

\section{Carousel Datum}

In order for the software to know the physical position of the nozzle carousel, a notch is cut into the wheel before the position of the first nozzle. An EE-SX4070 optical light gate is used with logic inversion in software, triggering a signal when the notch reaches the gate and the light is permitted to land on the phototransistor.

\section{Perspex Enclosure}

Surrounding the entire apparatus is an airtight perspex box (see Figure 7), with removable sides and gas entry and exit valves. This feature offers the ability to perform experiments in non-air environments, simply by flushing the air out of the box and introducing a different inert gas envelope. If the same gas is pre-bubbled through the sample prior to loading, this ensures the sample remains saturated with that gas, thereby facilitating experiments which investigate the role of the dissolved gas. This is an important factor in many circumstances; the production pathways of many water radiolytic species are dependent on oxygen content, for example. An additional benefit to the enclosed system is that the experiment is unaffected by exterior air disturbances, and if necessary a container of water can be placed inside the box to reduce sample evaporation. Along the beam axis, there are Kapton windows where the beam enters and exits the box, reducing the absorption of the beam. The perspex walls are contained to the footprint of the apparatus and do not extend beyond the dimensions stated previously (540 $\mathrm{mm} \times 330 \mathrm{~mm} \times 410 \mathrm{~mm}$ ).

\section{F. Software}

The software to operate the apparatus was designed and programmed in-house using National Instruments Labview 2012 suite. It interfaces with the hardware using 


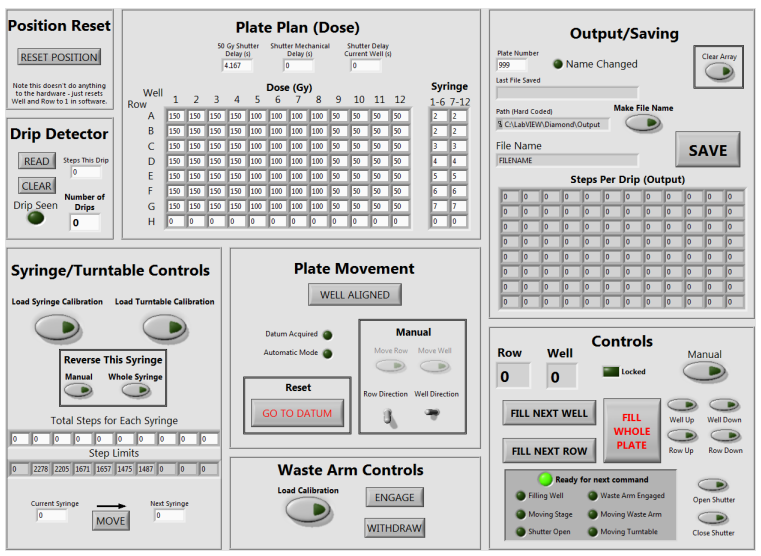

FIG. 8. The control panel, which allows the user to interact with the device.

\section{NI DAQ digital I/O drivers.}

The front control panel is where the user interfaces with the device (see Figure 8). It has several panels embedded within it to control different components of the hardware. The 'Plate Plan (Dose)' panel contains information for the next microplate of samples to be processed. The user can input the dose rate of the radiation source and the dose required for each droplet, as well as the syringe to be used for each half-row of samples. The same syringe is used for each half-row (6 samples). This is not a constraint of the system in any way, it was simply configured in this fashion for our application to reduce the amount of user input required. There is also an array which records the number of syringe motor steps before the droplet falls from the nozzle and triggers the detector, and outputs the table to a csv file for archival purposes. This is useful for cross referencing potentially anomalous results (droplet falling prematurely, small droplet etc.) although our experience is that these happen at less than the $0.1 \%$ level provided the system is well aligned.

While the operational protocol executes automatically according to the plate plan entered, it is also possible to operate components individually. There are controls to return the stage to the datum point and navigate the plate to any individual well, as well as to select syringes manually and express or withdraw them. The shutter can also be operated manually.

When a syringe is loaded with a material of a different density or viscosity, it is often necessary to calibrate the system for the new material properties to ensure consistent droplet size between samples. There is a separate protocol for this. Firstly, the syringe ejects a droplet to clear any dead space in the line, and then steps more slowly until another droplet falls. This gives a reasonable value of motor steps from droplet formation to dislodgement. Finally, the motor steps to $75 \%$ of this value before slowing down, and continues until the droplet falls. The slowing is to allow the droplet to stabilise in between steps, to obtain a more accurate value. This value is

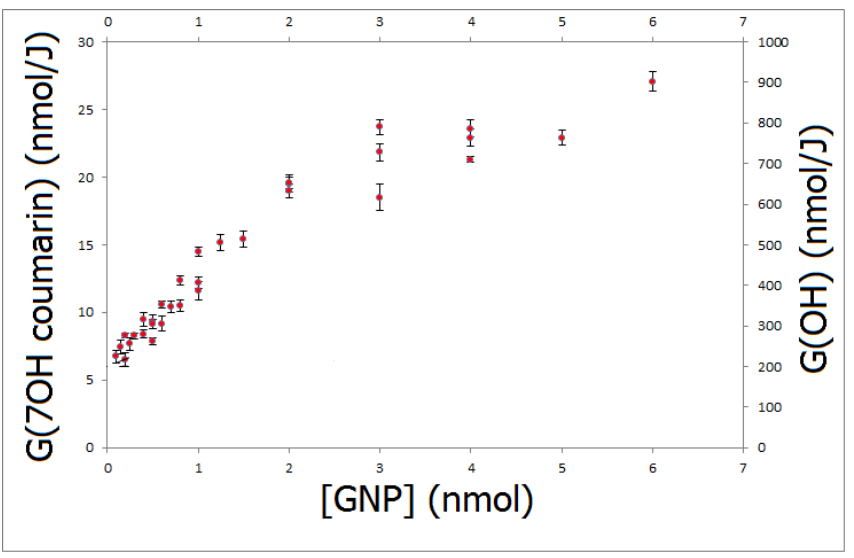

FIG. 9. The apparatus has been used extensively at the Diamond Light Source synchrotron to enable us to irradiate gold and silver colloid nanoparticle preparations. The nanoparticles are dissolved into a solution of water and Coumarin, which acts as a trapping assay for hydroxyl radical molecules produced via radiolysis. Figure 9 demonstrates the G-value of 7-OH Coumarin (a fluorescent compound formed when Coumarin interacts with hydroxyl radicals) as a function of GNP concentration. Figure from Sicard-Roselli et $a l^{5}$

recorded, to be read by the main protocol later when the syringe is in use.

\section{RESULTS}

An example of the device's use can be found in SicardRoselli $e t a l^{5}$, which details an experiment to investigate the radiolysis mechanisms which take place during the irradiation of a water and colloid gold solution; in particular, the influence the gold's presence has on the production of the hydroxyl radical. This is valuable information to the field of nanoparticle-based cancer therapeutics, as metallic nanoparticles have been shown to increase conventional radiotherapy efficacy. ${ }^{67}$ In this instance, the data analysis involved plotting the concentration of $\mathrm{OH}$ as a function of dose, the gradients of which were used to determine the $\mathrm{G}$-value (the number of molecules of a substance produced or destroyed at a given energy). This procedure was repeated for many different concentrations in order to determine the effect of the nanoparticles. All measurements were done in triplicate. Accordingly, a useful set of measurements required irradiation of many thousands of samples (see Figure 9). The apparatus described herein allowed this work to be carried out with relative ease and within realistic timescales for facility use where time may be limited. The droplets are expressed into a standard 96-well microplate which can then be removed and placed into a reader for easy analysis, and without further processing requirements for many colorimetric measurement protocols. 


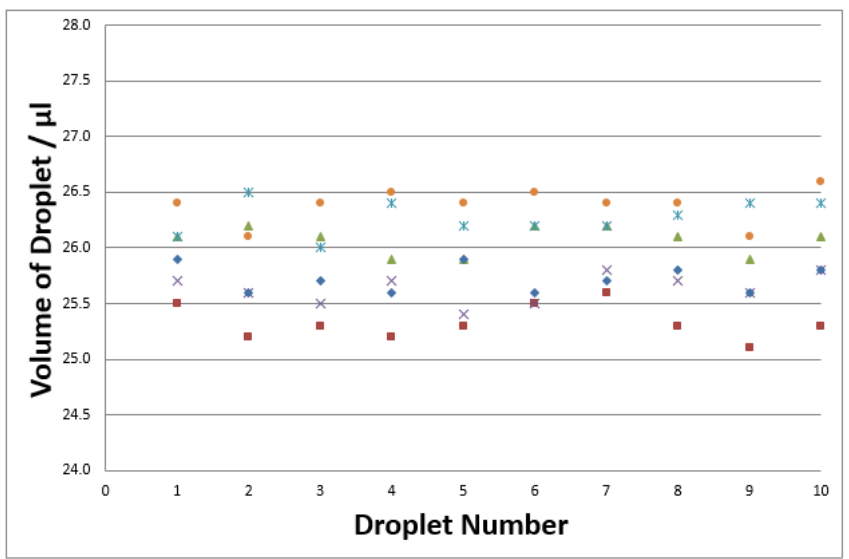

FIG. 10. This graph displays the consistency between droplets across a selection of five nozzles over ten droplets. The standard deviations for each nozzle across ten droplets in this characterisation study were all the same within the range $0.11 \mu l$ to $0.13 \mu l(0.4-0.5 \%$ deviation from the average droplet volume.). The overall average droplet volume was $25.1 \mu \mathrm{l}$, and the standard deviation for the average droplet volume per nozzle over the entire ten-syringe array was $0.5 \mu l$. $(2 \%)$

\section{A. Characterisation of Nozzles}

An example of the reproducibility of the droplet size is shown in Figure 10. Ten droplets of water were expressed and weighed to determine their volume, and the experiment was repeated across all ten nozzles in the apparatus. In other experiments samples of different concentrations of colloidal gold were used and similar results were obtained. However, comparing across the samples, a systematic variation of droplet size with sample density was observed. The data as a whole is best accounted for by treating the droplet mass as constant but in practice the range of densities we have used is small and this correction is negligible.

\section{B. Dosimetry}

As the doses entered into the plate plan are dependent on the dose rate of the incident beam, it is important to have a standard dosimetry protocol. The apparatus is designed to process liquid samples, therefore a Fricke dosimeter solution is a suitable method to determine dose rate. By loading one of the syringes with Fricke solution (0.4 M sulphuric acid, $6 \mathrm{mM}$ ammonium ferrous sulphate and $1 \mathrm{mM}$ potassium chloride $)^{8}$, the irradiation protocol is carried out as normal and the solution is analysed with a UV-Vis spectrometer.

\section{CONCLUSION}

In summary, the device described here is capable of high throughput automated liquid sample handling. It can process 96 samples (at a dose rate of $1 \mathrm{~Gy} / \mathrm{s}$ ) in little over 2 hours before human interaction is necessary. In a typical 72 hour experiment, including setup, plate changes and line refills, more than 2,000 droplets were expressed, each with a low volumetric error. Over half of this time was taken up irradiating samples.

\section{ACKNOWLEDGMENTS}

The authors would like to thank Martin McCullough and Warren McConnaughie in the mechanical workshop of the School of Mathematics and Physics, Queen's University Belfast for their valuable input, both in design and manufacture of the apparatus. Thanks go to Andrew Malandain, Slava Kachkanov, Allan Ross and Annette Kleppe at The Diamond Light Source for enabling us to use the device.

\section{REFERENCES}

${ }^{1}$ M. Misawa, J. Takahashi 'Generation of reactive oxygen species induced by gold nanoparticles under x-ray and UV Irradiations.' Nanomedicine : Nanotechnology, Biology, and Medicine (2011); Vol. 7; pp. 604-614

${ }^{2}$ R.J. Hilarides, K.A. Gray 'Innovative treatment of soil contamination: Radiolytic destruction of dioxin and co-contaminants by cobalt-60.' American Society of Civil Engineers (1994); Vol. 26; pp. $733-736$

${ }^{3} \mathrm{Z}$. Hai, et al 'Radiolytic synthesis of $\mathrm{CuS}$ nanotubes with photocatalytic activity under visible light.' Materials Letters (2013); Vol. 108; pp. 304-307

${ }^{4} \mathrm{X}$-ray Mass Attenuation Coefficient Database: www.physics.nist.gov;

${ }^{5}$ Sicard-Roselli, et al 'A New Mechanism for Hydroxyl Radical Production in Irradiated Nanoparticle Solutions.' Small (2014); Vol. 10; pp. 3338-3346

${ }^{6} \mathrm{~J}$. F. Hainfeld, et al 'The use of gold nanoparticles to enhance radiotherapy in mice.' Phys. Med. Biol. (2004); Vol. 49; pp. 309315

${ }^{7} \mathrm{~S}$. Jain, et al 'Cell-specific radiosensitization by gold nanoparticles at megavoltage radiation energies.' Int. J. Radiation Oncology Biol. Phys. (2011); Vol. 79; pp. 531-539

${ }^{8}$ J. W. T Spinks, R. J Woods 'Introduction to Radiation Chemistry (Third Edition)' Wiley New-York (1990) 